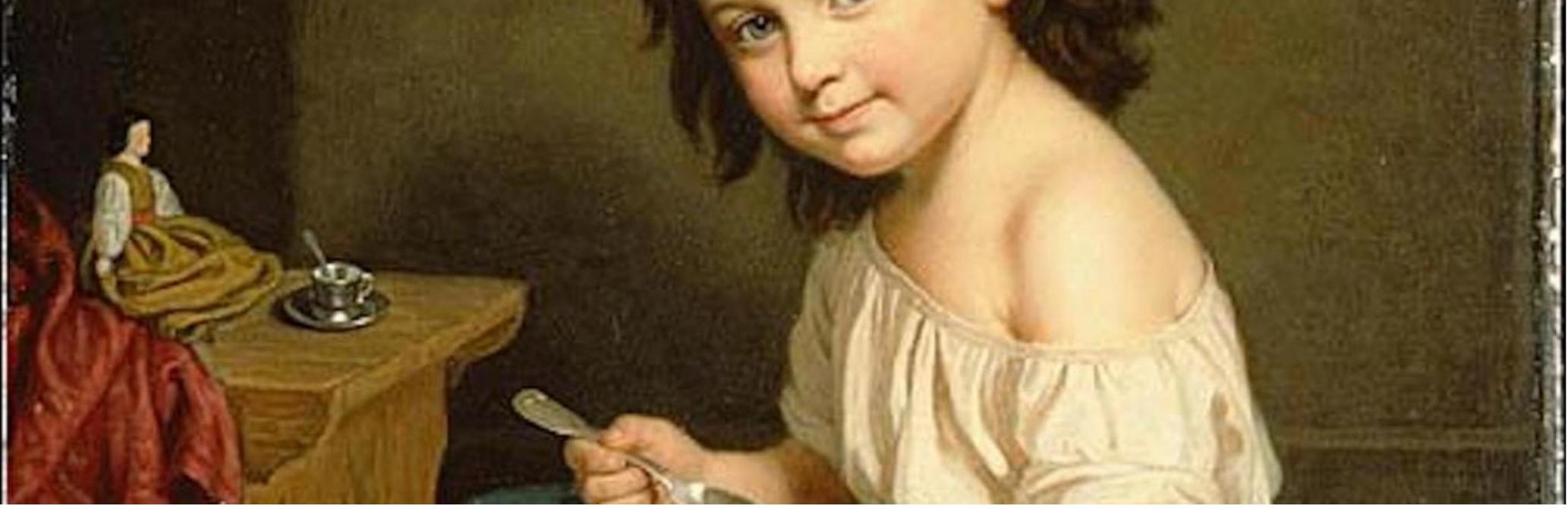

\title{
Urinary Tract Infection As A Predictor Of Poor Prognosis In Pediatric Patients With Severe Febrile Neutropenia Related To Chemotherapy
}

Authors: $\quad$ Maria Paula Gonzalez Galvis, Luis Alfonso Díaz-Martínez, Luis Miguel Sosa Ávila

$\begin{aligned} \text { Submitted: } & \text { 2. November } 2017 \\ \text { Published: } & \text { 2. November } 2017 \\ \text { Volume: } & 4 \\ \text { Issue: } & 5\end{aligned}$

Affiliation: Departamento de Pediatría, Escuela de Medicina, Universidad Industrial de Santander. Bucaramanga, Colombia

Keywords: $\quad$ Fever, Neutropenia, Pediatrics, Infection, Urine, Prognosis, Chemotherapy, Language: Spanish. Fiebre, Neutropenia, Pediatría, Infección, Orina, Pronóstico, Quimioterapia, Espanol DOI: $\quad$ 10.17160/josha.4.5.352 
Tipo de artículo: Artículo original

Especialidad: Pediatría

\title{
LA INFECCIÓN DE VÍAS URINARIAS COMO FACTOR DE MAL PRONÓSTICO EN PACIENTES PEDIÁTRICOS CON NEUTROPENIA FEBRIL SEVERA SECUNDARIA A QUIMIOTERAPIA
}

\author{
María Paula González Galvis* \\ Luis Alfonso Díaz-Martínez** \\ Luis Miguel Sosa Ávila*** \\ * Estudiante, Programa de Especialización en Pediatría, Departamento de Pediatría, Escuela \\ de Medicina, Universidad Industrial de Santander; actualmente Pediatra, Departamento de \\ Pediatría, Hospital Universitario de Santander, Bucaramanga, Colombia. \\ ** Profesor Titular. Escuela de Medicina, Universidad Industrial de Santander, Bucaramanga, \\ Colombia. \\ *** Profesor Asociado, Departamento de Pediatría, Escuela de Medicina, Universidad \\ Industrial de Santander, Bucaramanga, Colombia.
}

Correspondencia: María Paula González. Dirección: Departamento de Pediatría, Hospital Universitario de Santander, Carrera 33 No. 28- 126, décimo piso. Bucaramanga. Santander. Colombia. Correo electrónico: m-paulag@hotmail.com

Conflicto de interés: Ninguno

\section{RESUMEN}

Antecedentes: La pobre respuesta inflamatoria de los pacientes con neutropenia febril secundaria a quimioterapia hace que descartar una infección urinaria con solo evaluación de síntomas urinarios y examen de orina pueda generar falsos negativos, con serias consecuencias para estos pacientes. Objetivo: determinar si el examen de orina es predictor de alto riesgo de desarrollar complicaciones infecciosas graves en niños con neutropenia febril severa. Materiales y métodos: estudio de evaluación de tecnología diagnóstica con muestreo transversal de niños de 2 a 18 años con neutropenia febril severa asociada a quimioterapia. Se compararon los pacientes con diagnóstico o no de infección urinaria para estimar indicadores de desempeño diagnóstico del uroanálisis. Resultados: se captaron 107 episodios, 8 (16\%) con infección urinaria (3 con uroanálisis normal). Se halló con frecuencia nitritos, esterasas y piuria en los episodios con infección urinaria. La sensibilidad de todos los componentes del uroanálisis es baja, pero la capacidad predictiva positiva de todos ellos, solos o valorados en asociaciones, es tal, siendo mejor la de los nitritos positivos (valor predictivo positivo $100 \%$, IC $95 \% 83.5 \%$ a $100 \%$ ). Se presentó mayor incidencia de sepsis clínica, necesidad de $\mathrm{UCl}$ y letalidad en los pacientes con infección urinaria. Conclusión: el estudio inicial en este tipo de pacientes debe incluir uroanálisis y urocultivo, se debe considerar cualquier signo de inflamación en el uroanálisis como patológico, la infección urinaria es factor de mal pronóstico en el paciente con fiebre y neutropenia.

Palabras clave: fiebre, neutropenia, pediatría, infección, orina, pronóstico, quimioterapia. 


\section{URINARY TRACT INFECTION AS A PREDICTOR OF POOR PROGNOSIS IN PEDIATRIC PATIENTS WITH SEVERE FEBRILE NEUTROPENIA RELATED TO CHEMOTHERAPY}

\section{SUMMARY}

The diminish inflammatory response in patients with febrile neutropenia secondary to chemotherapy makes it hard to discard a urinary tract infection based solely on the physical evaluation of the patient and basic urine laboratory tests, this could lead to false negative results with serious consequences to the patients. Objective: To define if the urinary tract infection is a high-risk predictor of severe complications in the pediatric patient with febrile neutropenia-related with chemotherapy and to establish the diagnostic significance of normal urine sediment in the initial study. Methods: A Cross-sectional study of diagnostic technology. We included children with ages from 2 to 18 years old, with severe febrile neutropenia related to chemotherapy, we compared patients with and without urinary tract infection and evaluated poor prognosis outcomes and analyzed the diagnostic efficiency of urinalysis.

Results: We included 107 episodes, UTI (urinary tract infection) was diagnosed in 8 episodes $(16 \%)$, there was a higher frequency of nitrites, esterase and pyuria in the urinary tract infection episodes, $(p<0.001)$, three episodes of UTI had normal urinalysis, we found a low sensitivity for all components of urinalysis with greater specificity, NPV and LR + for all components alone or evaluated associations, with a better performance for nitrites. From the outcomes assessed we found more clinical sepsis, need of ICU (intensive care unit) and mortality in patients with urinary tract infection $(p=0.023, p=0.01$ y $p<0.001)$. Conclusion: The initial evaluation in this type of patient should include urinalysis and urine culture; we should consider any sign of inflammation in the urinalysis as pathologic; UTI is a bad prognosis factor in patients with fever and neutropenia.

Key words: fever, neutropenia, pediatrics, infection, urine, prognosis, chemotherapy.

\section{INTRODUCCIÓN}

La neutropenia febril inducida por quimioterapia en el paciente oncológico es una situación frecuente que puede producir complicaciones serias, generando una alta morbimortalidad (1). Por esta razón debe ser considerada una urgencia que amerita un estudio oportuno y completo para identificar el origen de la infección, e instaurar un tratamiento de forma rápida y dirigida (2).

El uso de una terapia antimicrobiana empírica de forma temprana ha mejorado el pronóstico del paciente neutropénico febril, con disminución de la morbimortalidad (2,3). Para poder realizar un manejo dirigido se crearon modelos predictivos o pronósticos con el fin de identificar aquellos pacientes que estarán en alto riesgo de tener complicaciones y así determinar la necesidad de antibioticoterapia, el tipo de antimicrobiano, la duración y ubicación (hospitalaria o ambulatoria) del tratamiento.

Entre los parámetros usados con más frecuencia para clasificar como alto o bajo riesgo, se encuentran los propuestos en el estudio realizado en el 2001 por Santolaya y colaboradores (4), el cual toma 5 factores evaluados al ingreso, identificados como los que mayor predicen 
una infección bacteriana invasiva y riesgo de complicaciones, estos son: Proteína $\mathrm{C}$ reactiva PCR $>90 \mathrm{mg} / \mathrm{dl}$, hipotensión, recaída de leucemia, plaquetas menores a 50.000 y menos de 7 días desde la última quimioterapia. Otros autores describen factores de riesgo adicionales como comorbilidades asociadas (5), neutropenia profunda y prolongada (6) y episodio de neutropenia febril intranosocomial, sin encontrar superioridad entre uno $\mathrm{u}$ otro predictor de riesgo (7).

Teniendo en cuenta estudios donde se señala que el tracto urinario es una fuente de infección importante en el paciente con neutropenia febril inducida por quimioterapia (8, 9); vemos necesario establecer la relación que existe entre la Infección de Vías Urinarias (IVU) y la probabilidad de desarrollar complicaciones médicas severas secundarias a la infección. En el caso del estudio de la infección del tracto urinario en este tipo de pacientes, en algunos protocolos se realiza solo examen general de orina y el urocultivo solo se recomienda en algunos casos como en presencia de síntomas urinarios, el resultado de un parcial de orina patológico o el uso de un catéter a nivel vesical intermitente o permanente $(3,10,11)$. Sin embargo, se ha evidenciado como a causa de la pobre respuesta inflamatoria con la que cursan este tipo de pacientes, los síntomas urinarios y la piuria pueden estar ausentes a pesar de cursar con una infección del tracto urinario comprobada por urocultivo $(9,10)$, razón por la cual es necesario determinar el significado diagnóstico del sedimento de orina normal y correlacionar los resultados del sedimento urinario con los resultados del uro cultivo en paciente pediátrico con neutropenia febril severa inducida por quimioterapia.

\section{METODOLOGÍA}

Este estudio es una evaluación de tecnología diagnóstica con muestreo transversal. El estudio fue aprobado previamente por el Comité Institucional de Ética de la Universidad Industrial de Santader y de la Clínica Materno infantil San Luis (CMISL). Los criterios de inclusión fueron tener entre 2 y 18 años de edad, control de esfínteres y diagnóstico de enfermedad oncológica en quimioterapia con un episodio de neutropenia febril severa; se excluyeron los episodios con urocultivo contaminado. Fueron captados entre Enero del 2013 y Diciembre del 2014 de los servicios de urgencias y hospitalización del Hospital Universitario de Santander y de la CMISL, Bucaramanga, Colombia.

Se tomaron al ingreso al estudio los exámenes rutinarios en paciente con neutropenia febril (cuadro hemático, PCR, hemocultivos), así como examen general de orina (EGO) y urocultivo por micción espontánea. El desenlace a predecir fue la existencia de IVU cuando se encontró urocultivo con mas de 100.000 Unidades Formadoras de Colonias (UFC) o gammagrafía que indicaba pielonefritis aguda en pacientes con urocultivo negativo $(12,13)$. Como desenlace secundario se consideraron muerte, requerimiento de cuidado intensivo o sepsis.

Se compararon los pacientes que tenían IVU frente a los que no con las pruebas $x^{2}, t$ de student o de Wilcoxon (umbral $\alpha<0.05$ ). La relación entre el sedimento urinario e IVU se realizó mediante indicadores de desempeño diagnóstico (sensibilidad, especificidad, valores predictivos y razones de verosimilitud) con sus intervalos de confianza del 95\% así como concordancia más allá del azar ( $\mathrm{k}$ media de Cohen). 


\section{RESULTADOS}

\section{Características de la población estudiada}

En los dos años de recolección se reclutaron 53 pacientes, quienes presentaron 107 episodios de neutropenia febril severa; 46 (86,8\%) pacientes tenían leucemia linfoide aguda, 4 (7,5\%) leucemia mieloide aguda y $3(5.7 \%)$ osteosarcoma. En $24(22.0 \%)$ de los episodios los pacientes tenían diagnóstico de una recaída de su enfermedad oncológica de base; en 27 $(25.0 \%)$ episodios los pacientes estaban hospitalizados y en $80(74.0 \%)$ consultaron por urgencia. Tres (3.8\%) pacientes tenían antecedente de infección de vías urinarias; ningún paciente tenía antecedente de cateterismo vesical o malformación del tracto urinario. En 59 $(55.0 \%)$ episodios los pacientes recibieron antibiótico en los siete días previos a la consulta (69.0\% profiláctico y $31.0 \%$ terapéutico para un proceso infeccioso previo). Cien (93.5\%) de los episodios correspondían a neutropenia febril de alto riesgo ya que cumplían uno o más criterios de severidad.

La frecuencia de los elementos del sedimento fue positivo en $13(12,1 \%)$ episodios: nitritos positivos $4(3,7 \%)$ episodios, esterasas positivas $5(4.6 \%)$ y piuria mayor a 5 leucocitos/campo en $13(12,1 \%)$ episodios; $3(2,8 \%)$ pacientes tenían los tres elementos positivos considerando piuria mayor a 5 células/campo. En 50 (46.7\%) episodios se identificó un proceso infeccioso, $13(12.2 \%)$ con más de un foco (Tabla 1); 13 (12.2\%) episodios fueron declarados también como con sepsis clínica, pero en tres no se pudo identificar un proceso infeccioso.

Tabla 1. Procesos infecciosos establecidos

\begin{tabular}{|l|c|c|c|}
\hline $\begin{array}{l}\text { Proceso } \\
\text { infeccioso }\end{array}$ & Episodios & $\begin{array}{c}\text { Porcentaje entre episodios } \\
\text { de neutropenia febril con } \\
\text { proceso infeccioso } \\
\text { identificado (n=50) }\end{array}$ & $\begin{array}{c}\text { Porcentaje entre } \\
\text { todos los } \\
\text { episodios de } \\
\text { neutropenia febril } \\
\text { (n=107) }\end{array}$ \\
\hline \multicolumn{3}{|c|}{ Procesos sistémicos } \\
\hline Bacteremia & 12 & $24.0 \%$ & $11.2 \%$ \\
\hline TBC diseminada & 1 & $2.0 \%$ & $0.9 \%$ \\
\hline Citomegalovirus & 1 & $2.0 \%$ & $0.9 \%$ \\
\hline & 15 & $30.0 \%$ & $14.0 \%$ \\
\hline ORL & 13 & $26.0 \%$ & $12.2 \%$ \\
\hline $\begin{array}{l}\text { Respiratorio } \\
\text { inferior }\end{array}$ & 8 & $16.0 \%$ & $7.8 \%$ \\
\hline Gastrointestinal & 8 & $16.0 \%$ & $7.8 \%$ \\
\hline Infección urinaria & 4 & $8.0 \%$ & $3.7 \%$ \\
\hline Piel y tejido blando & 1 & $2.0 \%$ & $0.9 \%$ \\
\hline Sitio de catéter & 1 & & \\
\hline
\end{tabular}


En las tablas 2 y 3 se reporta la frecuencia y el tipo de gérmenes aislados en los urocultivos y hemocultivos Se detectaron ocho episodios de infección urinaria, todos en pacientes diferentes: cinco por urocultivo positivo (dos $E$. coli, dos K. pneumoniae y uno $P$. aeruginosa) y tres detectados por imagenología luego que la evolución del paciente no fuese la esperada. Tres de los ocho episodios de IVU tenían un examen EGO normal (dos detectados por urocultivo y uno por imagenología). La letalidad de los cuadros de neutropenia febril severa estuvo representada en tres fallecimientos: $2,8 \%$ de los episodios y $5,7 \%$ de los pacientes.

Tabla 2. Resultado de los urocultivos positivos: microorganismo aislado y frecuencia

\begin{tabular}{|c|c|c|c|}
\hline $\begin{array}{c}\text { MICROORGANISMO } \\
\text { AISLADO }\end{array}$ & Episodios & $\begin{array}{c}\text { Porcentaje entre } \\
\text { episodios de } \\
\text { neutropenia febril } \\
\text { con microorganismo } \\
\text { aislado en } \\
\text { urocultivo (N=5) }\end{array}$ & $\begin{array}{c}\text { Porcentaje entre } \\
\text { episodios todos } \\
\text { los episodios de } \\
\text { neutropenia febril } \\
\text { (N=107) }\end{array}$ \\
\hline Escherichia coli & 2 & $40.0 \%$ & $1.9 \%$ \\
\hline $\begin{array}{c}\text { Klebsiella } \\
\text { pneumoniae }\end{array}$ & 2 & $40.0 \%$ & $1.9 \%$ \\
\hline $\begin{array}{c}\text { Pseudomonas } \\
\text { aeruginosa }\end{array}$ & 1 & $20.0 \%$ & $0.9 \%$ \\
\hline
\end{tabular}

Tabla 3. Resultado de los hemocultivos positivos: microorganismo aislado y frecuencia

\begin{tabular}{|l|c|c|c|}
\hline \multicolumn{1}{|c|}{$\begin{array}{c}\text { MICROORGANISMO } \\
\text { AISLADO }\end{array}$} & Episodios & $\begin{array}{c}\text { Porcentaje entre } \\
\text { episodios de } \\
\text { neutropenia febril con } \\
\text { microorganismo } \\
\text { aislado (N=12) }\end{array}$ & $\begin{array}{c}\text { Porcentaje entre } \\
\text { episodios todos los } \\
\text { episodios de } \\
\text { neutropenia febril } \\
\text { (N=107) }\end{array}$ \\
\hline Escherichia coli & 3 & $25.0 \%$ & $2.8 \%$ \\
\hline $\begin{array}{c}\text { Klebsiella } \\
\text { pneumoniae }\end{array}$ & 3 & $25.0 \%$ & $2.8 \%$ \\
\hline $\begin{array}{c}\text { Acinetobacter } \\
\text { baumannii }\end{array}$ & 2 & $16.6 \%$ & $1.7 \%$ \\
\hline Acinetobacter spp & 1 & $8.3 \%$ & $0.9 \%$ \\
\hline
\end{tabular}




\begin{tabular}{|c|c|c|c|}
\hline $\begin{array}{c}\text { Pseudomonas } \\
\text { aeruginosa }\end{array}$ & 1 & $8.3 \%$ & $0.9 \%$ \\
\hline $\begin{array}{c}\text { Staphylococcus } \\
\text { saprophyticus }\end{array}$ & 1 & $8.3 \%$ & $0.9 \%$ \\
\hline $\begin{array}{c}\text { Streptococcus } \\
\text { mitis }\end{array}$ & 1 & $8.3 \%$ & $0.9 \%$ \\
\hline
\end{tabular}

Factores asociados con la presentación de infección urinaria

Los cuadros de infección urinaria son más frecuentes en las niñas, pero no se encontraron diferencias en cuanto a edad, procedencia, patología oncológica de base, estar hospitalizados, estar en recaída y el uso de antibióticos previo al estudio (Tablas 4 y 5 ).

Tabla 4. Características demográficas y clínicas de los pacientes según presencia o no de infección urinaria

\begin{tabular}{|c|c|c|c|}
\hline \multirow[t]{2}{*}{ Característica } & \multicolumn{2}{|c|}{$\begin{array}{c}\text { Infección de la vía } \\
\text { urinaria }\end{array}$} & \multirow[t]{2}{*}{ Estadístico } \\
\hline & Si $(n=8)$ & No $(n=50)$ & \\
\hline Edad (años)* $^{*}$ & $5.5(3.5-13.0)$ & $6.0(4.0-9.0)$ & $\begin{array}{c}Z=-0.212 \\
p=0.832\end{array}$ \\
\hline $\begin{array}{l}\text { Sexo } \\
\text { Masculino } \\
\text { Femenino }\end{array}$ & $\begin{array}{l}1(12.5 \%) \\
7(87.5 \%)\end{array}$ & $\begin{array}{l}27(60.0 \%) \\
18(40.0 \%)\end{array}$ & $\begin{array}{c}X^{2}=0.150,1 \mathrm{gl}, \\
p=0.013\end{array}$ \\
\hline $\begin{array}{l}\text { Residencia } \\
\text { Bucaramanga } \\
\text { Otros Santander } \\
\text { Otros departamentos }\end{array}$ & $\begin{array}{l}5(62.5 \%) \\
2(25.0 \%) \\
1(12.5 \%)\end{array}$ & $\begin{array}{l}22(48.9 \%) \\
8(17.8 \%) \\
15(33.3 \%)\end{array}$ & $\begin{array}{c}\mathrm{X}^{2}=1.411,2 \mathrm{gl}, \\
\mathrm{p}=0.494\end{array}$ \\
\hline $\begin{array}{l}\text { Diagnóstico } \\
\text { Leucemia linfoide aguda } \\
\text { Leucemia mieloide aguda } \\
\text { Osteosarcoma }\end{array}$ & $\begin{array}{l}6(75.0 \%) \\
1(12.5 \%) \\
1(12.5 \%)\end{array}$ & $\begin{array}{c}40(88.9 \%) \\
3(6.7 \%) \\
2(4.4 \%)\end{array}$ & $\begin{array}{c}\mathrm{X}^{2}=1.236,2 \mathrm{gl}, \\
\mathrm{p}=0.539\end{array}$ \\
\hline
\end{tabular}

${ }^{*}$ Presentado como mediana y RIQ 
Tabla 5. Antecedentes clínicos de los episodios de neutropenia febril severa según presencia o no de infección urinaria

\begin{tabular}{|c|c|c|c|}
\hline \multirow[t]{2}{*}{ Característica } & \multicolumn{2}{|c|}{$\begin{array}{c}\text { Infección de la vía } \\
\text { urinaria }\end{array}$} & \multirow[t]{2}{*}{ Estadístico } \\
\hline & Si $(n=8)$ & No $(n=99)$ & \\
\hline Historia de IVU previa & $1(12.5 \%)$ & $1(2.2 \%)$ & $\begin{array}{c}\mathrm{X}^{2}=1.976,2 \mathrm{gl} \\
\mathrm{p}=0.160\end{array}$ \\
\hline Estaba hospitalizado & $1(12.5 \%)$ & $26(26.3 \%)$ & $\begin{array}{c}\mathrm{X}^{2}=0.743,1 \mathrm{gl}, \\
p=0.389\end{array}$ \\
\hline Estaba en recaída & $3(37.5 \%)$ & $21(21.2 \%)$ & $\begin{array}{c}\mathrm{X}^{2}=1.139,1 \mathrm{gl} \\
\mathrm{p}=0.288\end{array}$ \\
\hline $\begin{array}{l}\text { Tiempo de latencia } \\
\text { (días)* }^{*}\end{array}$ & $2.5(0.0$ a 6.5$)$ & $\begin{array}{c}6.0(3.0 \mathrm{a} \\
11.0)\end{array}$ & $Z=1.474 ; p=0.141$ \\
\hline Antibiótico previo $<1$ sem & $4(50.0 \%)$ & $55(55.6 \%)$ & $\begin{array}{c}\mathrm{X}^{2}=0.092,1 \mathrm{gl}, \\
p=0.761\end{array}$ \\
\hline $\begin{array}{l}\text { Antibiótico previo } \\
\text { terapéutico }\end{array}$ & $3(37.5 \%)$ & $15(15.2 \%)$ & $\begin{array}{c}\mathrm{x}^{2}=3.753,2 \mathrm{gl} \\
\mathrm{p}=0.153\end{array}$ \\
\hline
\end{tabular}

${ }^{*}$ Presentado como mediana y RIQ

Es más frecuente que los pacientes con infección urinaria y neutropenia febril manifiesten disuria; sin embargo, no se encontraron diferencias entre los demás síntomas irritativos del tracto urinario como tenesmo, pujo y frecuencia urinaria. No se encontró asociación entre los parámetros de laboratorio como neutrófilos absolutos al ingreso del estudio, Proteína $\mathrm{C}$ Reactiva inicial y su variación durante el seguimiento, así como el recuento plaquetario. La mayoría de los pacientes cumplían criterios de neutropenia febril de alto riesgo al ingreso al estudio, pero no se encontraron diferencias en cuanto a este parámetro entre los pacientes con infección de vías urinarias y los que no la presentaban (Tabla 6). 
Tabla 6. Características clínicas y de laboratorio de los episodios de neutropenia febril severa según presencia o no de infección urinaria

\begin{tabular}{|c|c|c|c|}
\hline \multirow[t]{2}{*}{ Característica } & \multicolumn{2}{|c|}{ Infección de la vía urinaria } & \multirow[t]{2}{*}{ Estadístico } \\
\hline & $S i(n=8)$ & No $(n=99)$ & \\
\hline Días de fiebre* & $1.0(1.0$ a 1.0$)$ & $1.0(1.0$ a 2.0$)$ & $Z=0.862 ; p=0.389$ \\
\hline Disuria & $2(25.0 \%)$ & $1(1.0 \%)$ & $\begin{array}{c}X^{2}=15.632,1 \mathrm{gl}, \\
p<0.001\end{array}$ \\
\hline Temperatura $\left({ }^{\circ} \mathrm{C}\right)^{*}$ & $\begin{array}{c}38.0 \\
(38.0 \text { a } 38.5)\end{array}$ & $\begin{array}{c}38.5 \\
(38.1 \text { a } 39.0)\end{array}$ & $Z=1.842 ; p=0.065$ \\
\hline $\begin{array}{l}\text { Llenado capilar } \\
\text { Aumentado } \\
\text { Normal } \\
\text { Retrasado }\end{array}$ & $\begin{array}{c}- \\
7(87.5 \%) \\
1(12.5 \%)\end{array}$ & $\begin{array}{c}2(2.0 \%) \\
93(93.9 \%) \\
4(4.0 \%)\end{array}$ & $\begin{array}{c}\mathrm{X}^{2}=1.328,2 \mathrm{gl} \\
\mathrm{p}=0.515\end{array}$ \\
\hline Neutrófilos absolutos* & $74(23$ a 261$)$ & $92(19$ a 280$)$ & $Z=0.036 ; p=0.972$ \\
\hline PCR inicial $(\mathrm{mg} / \mathrm{dL})^{*}$ & $\begin{array}{c}90.0 \\
(38.5 \text { a } 154.5)\end{array}$ & $\begin{array}{c}51,0 \\
(24.0 \text { a } 110.0)\end{array}$ & $Z=-0.926 ; p=0.354$ \\
\hline Plaquetas $\left(\text { por } \mathrm{mm}^{3}\right)^{*}$ & $\begin{array}{c}42550 \\
(10485 a \\
101000)\end{array}$ & $\begin{array}{c}34000 \\
(11000 a \\
93100)\end{array}$ & $Z=-0.136 ; p=0.892$ \\
\hline $\begin{array}{l}\text { Neutropenia febril de alto } \\
\text { riesgo }\end{array}$ & $7(87.5 \%)$ & $93(93.9 \%)$ & $\begin{array}{l}X^{2}=0.502,2 \mathrm{gl}, \\
p=0.479\end{array}$ \\
\hline Nitritos & $4(50.0 \%)$ & - & $\begin{array}{c}X^{2}=51.422,1 \mathrm{gl}, \\
p<0.001\end{array}$ \\
\hline Esterasas & $3(37.5 \%)$ & $2(2.0 \%)$ & $\begin{array}{c}X^{2}=20.917,1 \mathrm{gl}, \\
p<0.001\end{array}$ \\
\hline Leucocituria & & & \\
\hline 0-5 por campo & $3(37.5 \%)$ & $91(91.9 \%)$ & $X^{2}=25.263,3 \mathrm{gl}$ \\
\hline 6-10 por campo & $2(25.0 \%)$ & $4(4.0 \%)$ & $p<0.001$ \\
\hline $11-20$ por campo & $1(12.5 \%)$ & $3(3.0 \%)$ & \\
\hline $21+$ por campo & $2(25.0 \%)$ & $1(1.0 \%)$ & \\
\hline Piuria $>5$ por campo & $5(62.5 \%)$ & $8(8.1 \%)$ & $\begin{array}{c}X^{2}=20.847,1 \mathrm{gl}, \\
p<0.001\end{array}$ \\
\hline Piuria>10 por campo & $3(37.5 \%)$ & $3(4.0 \%)$ & $\begin{array}{c}X^{2}=13.554,1 \mathrm{gl}, \\
p<0.001\end{array}$ \\
\hline
\end{tabular}

${ }^{*}$ Presentado como mediana y RIQ 
Se presentaron más episodios con sepsis clínica entre los pacientes con infección de vías urinarias versus aquellos sin infección con una diferencia significativa; de la misma forma fue mayor la necesidad de $\mathrm{UCl}$ en este grupo de pacientes, así como la letalidad (Tabla 7).

Tabla 7. Desenlaces clínicos de los episodios de neutropenia febril severa según si tenían o no infección urinaria.

\begin{tabular}{|l|c|c|c|}
\hline \multicolumn{1}{|c|}{ Característica } & \multicolumn{2}{|c|}{ Infección de la vía urinaria } & \multirow{2}{*}{ Estadístico } \\
\cline { 2 - 3 } & $\mathbf{S i}(\mathbf{n}=\mathbf{8})$ & No $(\mathbf{n}=\mathbf{9 9})$ & \\
\hline Sepsis clínica & $3(37.5 \%)$ & $10(10.1 \%)$ & $\begin{array}{c}\mathrm{X}^{2}=5.201,1 \mathrm{gl}, \\
\mathrm{p}=0.023\end{array}$ \\
\hline Necesidad de UCl & $3(37.5 \%)$ & $5(5.1 \%)$ & $\begin{array}{c}\mathrm{X}^{2}=11.267,1 \\
\mathrm{gl}, \mathrm{p}=0.001\end{array}$ \\
\hline Letalidad & $2(25.0 \%)$ & $1(1.0 \%)$ & $\begin{array}{c}\mathrm{X}^{2}=15.63,1 \mathrm{gl}, \\
\mathrm{p}<0.001\end{array}$ \\
\hline
\end{tabular}

${ }^{*}$ Presentado como mediana y RIQ

En la tabla 8 se hallan los indicadores diagnósticos de cada componente del sedimento urinario, solos o en diversas combinaciones. En general, tienen una alta especificidad con baja sensibilidad, los cuales van de la mano con valor predictivo y razón de verosimilitud (LR) negativo bajo y valor predictivo y LR positivo alto, siendo mejor el comportamiento para nitritos y esterasa que para piuria. Todos los elementos de sedimento presentaron con una concordancia más allá del azar adecuada. 
Tabla 8. Indicadores diagnósticos de cada componente positivo del uroanálisis en el diagnóstico de IVU en pacientes con neutropenia febril severa

\begin{tabular}{|c|c|c|c|c|c|c|c|c|c|c|c|c|c|c|}
\hline \multirow{3}{*}{$\begin{array}{l}\text { Pruebal } \\
\text { Indicad } \\
\text { or }\end{array}$} & \multicolumn{2}{|c|}{ Nitritos } & \multicolumn{2}{|c|}{ Esterasa } & \multicolumn{2}{|c|}{ Piuria $>5$} & \multicolumn{2}{|c|}{$\begin{array}{l}\text { Nitritos y } \\
\text { Esterasa }\end{array}$} & \multicolumn{2}{|c|}{$\begin{array}{c}\text { Nitritos y } \\
\text { Piuria }>5\end{array}$} & \multicolumn{2}{|c|}{$\begin{array}{l}\text { Nitritos o } \\
\text { Esterasa }\end{array}$} & \multicolumn{2}{|c|}{$\begin{array}{c}\text { Nitritos } 0 \\
\text { Piuria }>5\end{array}$} \\
\hline & \multicolumn{2}{|c|}{ IVU } & \multicolumn{2}{|c|}{ IVU } & \multicolumn{2}{|c|}{ IVU } & \multicolumn{2}{|c|}{ IVU } & \multicolumn{2}{|c|}{ IVU } & \multicolumn{2}{|c|}{ IVU } & \multicolumn{2}{|c|}{ IVU } \\
\hline & $\mathrm{Si}$ & No & $\mathrm{Si}$ & No & $\mathrm{Si}$ & $\mathrm{Si}$ & No & $\mathrm{Si}$ & No & No & $\mathrm{Si}$ & No & Si & No \\
\hline Posi & 4 & - & 3 & 2 & 5 & 4 & 2 & 4 & 2 & 4 & 4 & 2 & 5 & 8 \\
\hline $\begin{array}{l}\text { Negativ } \\
\text { o }\end{array}$ & 4 & 99 & 5 & 97 & 3 & 4 & 97 & 4 & 97 & 95 & 4 & 97 & 3 & 91 \\
\hline $\begin{array}{l}\text { Sensibili } \\
\text { dad }\end{array}$ & \multicolumn{2}{|c|}{$\begin{array}{c}50.0(9.1- \\
90.9)\end{array}$} & \multicolumn{2}{|c|}{$\begin{array}{c}37.5(0.0- \\
77.3)\end{array}$} & \multicolumn{2}{|c|}{$\begin{array}{c}62.5(22.7- \\
100)\end{array}$} & \multicolumn{2}{|c|}{$\begin{array}{c}37.5(0.0- \\
77.3)\end{array}$} & \multicolumn{2}{|c|}{$\begin{array}{c}50.0(9.1- \\
90.9)\end{array}$} & \multicolumn{2}{|c|}{$\begin{array}{c}50.0(9.1- \\
90.9)\end{array}$} & \multicolumn{2}{|c|}{$\begin{array}{c}62.5(22.7- \\
100)\end{array}$} \\
\hline $\begin{array}{l}\text { Especifi } \\
\text { cidad }\end{array}$ & \multicolumn{2}{|c|}{$\begin{array}{c}100(99.5- \\
100)\end{array}$} & \multicolumn{2}{|c|}{$\begin{array}{c}98.0(94.7- \\
100)\end{array}$} & \multicolumn{2}{|c|}{$\begin{array}{c}91.9(86.1- \\
97.8)\end{array}$} & \multicolumn{2}{|c|}{$\begin{array}{c}100(99.5- \\
100)\end{array}$} & \multicolumn{2}{|c|}{$\begin{array}{c}100(99.5- \\
100)\end{array}$} & \multicolumn{2}{|c|}{$\begin{array}{c}98.0(94.7- \\
100)\end{array}$} & \multicolumn{2}{|c|}{$\begin{array}{c}91.9(86.1- \\
97.8)\end{array}$} \\
\hline VPP & \multicolumn{2}{|c|}{$\begin{array}{c}100(83.5- \\
100)\end{array}$} & \multicolumn{2}{|c|}{$\begin{array}{c}60.0(7.1- \\
100)\end{array}$} & \multicolumn{2}{|c|}{$\begin{array}{c}33.5(8.2- \\
68.8)\end{array}$} & \multicolumn{2}{|c|}{$\begin{array}{c}100(83.3- \\
100)\end{array}$} & \multicolumn{2}{|c|}{$\begin{array}{c}100(83.5- \\
100)\end{array}$} & \multicolumn{2}{|c|}{$\begin{array}{c}66.7(20.6- \\
100)\end{array}$} & \multicolumn{2}{|c|}{$\begin{array}{c}38.5(8.2- \\
68.8)\end{array}$} \\
\hline VPN & & & $\begin{array}{r}95.1 \\
9 \\
\end{array}$ & & & & $\begin{array}{r}95.2 \\
9 \\
\end{array}$ & & & & & & & \\
\hline LR+ & Inde & inido & & & & & Inde & nido & Inde & nido & & & & \\
\hline LR- & $\begin{array}{r}0 \\
(0.25 \\
\end{array}$ & $\begin{array}{l}0 \\
1.00)\end{array}$ & $\begin{array}{r}0 \\
(0.37 \\
\end{array}$ & $\begin{array}{l}4 \\
1.09)\end{array}$ & $\begin{array}{r}0 \\
(0.17 \\
\end{array}$ & $\begin{array}{l}1 \\
1.00) \\
\end{array}$ & $\begin{array}{r}0 \\
(0.37 \\
\end{array}$ & $1.07)$ & $\begin{array}{r}0 \\
(0.25 \\
\end{array}$ & $\begin{array}{l}0 \\
1.00)\end{array}$ & $(0.26$ & $\begin{array}{l}51 \\
1.02)\end{array}$ & $(0.17$ & $\begin{array}{l}1 \\
1.00)\end{array}$ \\
\hline Kappa & & & & & & & & & & & & & & \\
\hline
\end{tabular}

VPP: Valor predictivo positivo

VPN: Valor predictivo negativo

LR+: Razón de verosimilitud positiva

LR-: Razón de verosimilitud negativa

\section{DISCUSIÓN}

Según lo reportado en la literatura, la neutropenia febril se presenta más en pacientes con neoplasias hematolinfoides $(3,12)$. En nuestro estudio el $94,3 \%$ de los episodios correspondían a leucemias linfoides o mieloides, lo cual podría explicarse debido al uso de quimioterapia más agresiva en este tipo de neoplasias. De los episodios de neutropenia febril severa incluidos en el estudio, tres cuartas partes fueron iniciados cuando el paciente estaba en casa, lográndose definir un foco infeccioso en cerca de la mitad de los casos en una 
proporción similar a lo reportado en la literatura $(13,14)$; las IVU fueron el cuarto grupo en frecuencia.

Es de resaltar que la frecuencia de infección de vías urinarias en nuestro estudio es mayor a la alcanzada en reportes locales, como en el estudio de Rueda y colaboradores (12), donde se reporta una frecuencia de $2.5 \%$. Sin embargo, en diferentes trabajos internacionales se ha identificado la infección urinaria como un foco importante, con una prevalencia que varía desde el $10 \%$ al $26 \%(8,9,15-17)$, lo cual se ajusta a lo encontrado en el presente trabajo; esto implica que al ser un foco de infección importante debe realizarse una búsqueda activa del mismo.

La infección de vías urinarias en nuestro estudio fue más frecuente en el género femenino, acorde a los datos encontrados de infección de vías urinarias en la población general $(18,19)$, esto indica que, a pesar de su condición de inmunosupresión, no hay un aumento de riesgo por género de sufrir IVU. No obstante, en el paciente inmunosuprimido la Escherichia coli sigue siendo el principal patógeno causante de infección de vías urinarias, seguido por Klebsiella pneumonie y Enterococcus faecalis (8), similar a los observado en nuestro trabajo.

Una década atrás la letalidad del paciente pediátrico con neutropenia febril era del $10 \%$; actualmente se reporta una frecuencia que oscila entre $2 \%$ al $6 \%(4,10)$, lo cual evidencia el impacto favorable del diagnóstico oportuno y el manejo adecuado. En nuestro estudio la letalidad fue de 3 casos (5,7\% de los pacientes), lo que a pesar de correlacionarse con las estadísticas reportadas en otras revisiones, sigue siendo sustancial y necesita de una mejor aproximación.

En cuanto al abordaje diagnóstico de la infección de vías urinarias en el paciente con neutropenia febril severa secundaria a quimioterapia, las guías indican que solo es necesario realizar urocultivo en caso de que presente algún factor de riesgo como infección urinaria recurrente, malformación de tracto urinario o cateterismo vesical, así como a los que tengan alguna manifestación sintomática del tracto urinario o tengan un examen de orina patológico $(3,11,20)$. Sin embargo, en nuestro estudio la mayoría de los pacientes con IVU no tenían ningún antecedente (solo uno tenía historia de IVU en eventos previos) por lo que no debería ser tomado este aspecto como único predictor de esta infección.

Diferentes reportes indican que la infección de vías urinarias en el paciente con neutropenia febril puede presentarse con síntomas inespecíficos, en algunos casos manifestándose solo con fiebre (21). En el caso de nuestro estudio, la mayoría de los pacientes con IVU presentaban disuria. Es de resaltar que a los diagnosticados por gammagrafía no presentaban estas manifestaciones; teniendo en cuenta esto, los síntomas urinarios deben ser tomados como base para el estudio exhaustivo de este foco, sin embargo, no deben ser usados para descartar esta infección en caso de su ausencia.

Tres de los ocho episodios tenían un examen de orina completamente normal y en quienes la detección de la infección urinaria se hizo por urocultivo (dos pacientes) y otro por imagenología. Este hallazgo indica que un uroanálisis normal no es suficiente para descartar la infección urinaria durante un episodio de neutropenia (21), razón por la cual debe continuarse utilizando el urocultivo en la evaluación inicial de este tipo de pacientes. Así, solo 
cinco de los ocho episodios fueron detectados por anomalías en el EGO y urocultivo, lo que indica la necesidad de buscar este foco de infección por medio de búsqueda activa más allá de la valoración regular sino con seguimiento estricto en pacientes que no responden como se espera, sobre todo si se tiene en cuenta que tienen mayor riesgo de muerte 0 complicaciones severas (19).

Al analizar la validez diagnostica de cada uno de los componentes (nitritos, esterasa y piuria), se encuentra una baja sensibilidad para todos, lo cual indica que el examen general de orina no puede ser usado como método de tamizaje en estos pacientes ya que no permite descartar con certeza a quienes no cursan con IVU, razón por la cual debe continuarse utilizando el urocultivo en la evaluación inicial de este tipo de pacientes.

En el caso de la esterasa y la piuria, los indicadores de validez encontrados en el presente estudio ya sean solos o en asociación con los demás parámetros, indican que estos ayudarían a discriminar los pacientes que probablemente sí tienen la infección, pero sin poder confirmarla, y su negatividad no excluiría la enfermedad, contrario a lo evidenciado en estudios de la población general $(18,19)$. Este hallazgo puede ser explicado por la falta de respuesta inflamatoria adecuada ante una infiltración bacteriana y a la leucopenia encontrada en estos pacientes, lo cual limitaría el número de leucocitos disponibles para ser excretados en la orina, aun en la presencia de una infección local $(9,21)$.

La mayoría de los pacientes con infección de vías urinarias cumplían criterios de alto riesgo al ingreso al estudio, sin embargo no se encontraron diferencias significativas en este parámetro entre los pacientes con IVU y los que no la presentaban. Como hallazgo importante evidenciamos una mayor cantidad de episodios con sepsis clínica, necesidad de UCl y una mayor letalidad en los pacientes con infección de vías urinarias; esto indicaría que los últimos tres desenlaces evaluados estarían relacionados directamente con presentar una infección de vías urinarias, implicando que el tener este diagnóstico debe ser valorado como un signo de alarma el cual predice una evolución más tórpida y mayor posibilidad de presentar complicaciones.

Como limitaciones se tiene que en $55 \%$ de los episodios los pacientes habían recibido antibiótico antes del ingreso al estudio, lo cual podría disminuir la probabilidad de obtener un urocultivo positivo; a pesar de esto, no se presentaron diferencias significativas entre los episodios con infección de vías urinarias y los que no la presentaban.

Es probable que el bajo número de episodios con infección de vías urinarias le reste precisión al estudio, lo cual pudo verse reflejado en los intervalos de confianza amplios de los indicadores de desempeño diagnostico; sin embargo, esto no le resta poder al estudio ya que se lograron establecer diferencias. Potencialmente, puede que haya un sesgo de selección que es imposible de estimar, dados por ciertos pacientes que conocemos se perdieron siendo susceptibles de ser elegidos, debido a que solo se realizó urocultivo o uroanálisis y no los dos en conjunto por lo que no se pudieron comparar. 


\section{CONCLUSIONES}

A manera de conclusión, la infección de vías urinarias es frecuente en pacientes con neutropenia febril severa asociada a quimioterapia y lleva a una alta morbimortalidad, razón por la que debe investigarse de forma exhaustiva en todos los casos; el estudio debe incluir examen de orina y urocultivo de forma inicial, ya que los resultados de los diferentes componentes evaluados en el uroanalisis (nitritos, esterasa y piuria) ayudarían a detectar la infección, más no a descartarla. Recomendamos continuar la evaluación de una posible infección urinaria en aquellos pacientes con una evolución tórpida que tengan algún dato ya sea clínico (ej. síntomas irritativos, puño percusión positiva) paraclínico (uro análisis inicial anormal) o antecedente relacionado con mayor riesgo de infección urinaria, a pesar que el urocultivo inicial sea negativo. Se necesitan estudios adicionales evaluando este tipo de pacientes que presentan evolución tórpida con gammagrafia renal para confirmar esta recomendación. 


\section{REFERENCIAS}

1. Zhi-Yong KE, Ling XU. A prospective study of febrile episodes in inpatient children on chemotherapy, Pediatr Infect Dis J 2010; 29:968-70.

2. Phillips RS, Sutton AJ. Predicting infectious complications in neutropenic children and young people with cancer. Systematic Rev 2012; 1:2-10.

3. Freifeld AG, Bow EJ, Sepkowitz K. Clinical practice guideline for the use of antimicrobial agents in neutropenic patients with cancer. Clinical Infectious Diseases 2011; 52:e56-e93.

4. Santolaya ME, Álvarez AM, Becker A, Cofré J. Prospective, multicenter evaluation of risk factors associated with invasive bacterial infection in children with cancer, neutropenia, and fever. J Clin Oncol 2001; 19:15-21.

5. Del Pont JM, Casanueva E. Comité Nacional de Infectología Pediátrica. Consenso sobre el cuidado del paciente oncológico neutropénico febril. Arch Argent Pediatr 2010; 9:47-70.

6. Dubos, F., Delebarre, M. Predicting the risk of severe infection in children with chemotherapy-induced febrile neutropenia. Curr Op Hematology 2012; 19:39-43.

7. Martinez AM, Perez JM. Procalcitonin and C-Reactive Protein serum levels as markers of infection in a pediatric population with febrile neutropenia and cancer. Pediatr Hematol Oncol 2009; 26:414-25.

8. Bakhshi S, Padmanjali KS, Arya LS. Infections in childhood acute lymphoblastic leukemia: an analysis of 222 febrile neutropenic episodes. Pediatr Hematol Oncol 2008, 25:385-92.

9. Klaassen IL, Bijlsma M, Bo A. Pyuria is absent during urinary tract infections in neutropenic patients. Pediatr Blood Cancer 2011; 56:868-70.

10. Uribe A. Clinical features of the neutropenic host: definitions and initial evaluation. Clin Infect Dis 2004; 39:53-5.

11. Jaramillo AC, Valencia MI, Aristizábal M. Neutropenia febril en pacientes pediátricos: un enfoque diagnóstico y terapéutico. latreia 2009; 22:235-45.

12. Rueda E, Trujillo M, Díaz LA. La neutropenia severa febril en niños con cáncer. Estudio descriptivo en el Hospital Universitario de Santander, Salud UIS 2010; 42:103-11.

13. Ramírez L, Blanco N, Hernández M. Episodios de neutropenia febril en niños con neoplasias malignas. Infectio 2003; 7:137-46.

14. Santolaya M, Rabagliati R, Bidart T. Consenso Manejo racional del paciente con cáncer, neutropenia y fiebre. Rev Chil Infect 2005; 22:79-113

15. Tezcan G, Kupesiz A, Ozturk F. Episodes of fever and neutropenia in children with cancer in a tertiary care medical center in Turkey. Pediatr Hematol Oncol 2006; 23:217-29.

16. Gupta S, Bonilla M, Gamero M. Microbiology and mortality of pediatric febrile neutropenia. J Pediatr Hematol Oncol 2011; 33:276-80.

17. Shankar SM, Nania JJ. Management of Pneumocystis jiroveci pneumonia in children receiving chemotherapy. Paediatr Drugs. 2007; 9:301-9.

18. Urinary tract infection in children: diagnosis, treatment and long term management, United Kingdom's National Institute for Health and Clinical Excellence (NICE) guideline on UTI, 2007. 
19. Newman TB. The new American Academy of Pediatrics urinary tract infection guideline. Pediatrics 2011; 128:572-5.

20. Matloob M, Fadoo Z. Febrile neutropenia in pediatric cancer patients: Experience from a tertiary health care facility of Pakistan. Pediatr Infect Dis 2014, 6:89-93.

21. Sandoval C, Sinaki B, Weiss S. Urinary tract infections in pediatric oncology patients with fever and neutropenia. Pediatr Hematol Oncol 2012; 29:68-72. 